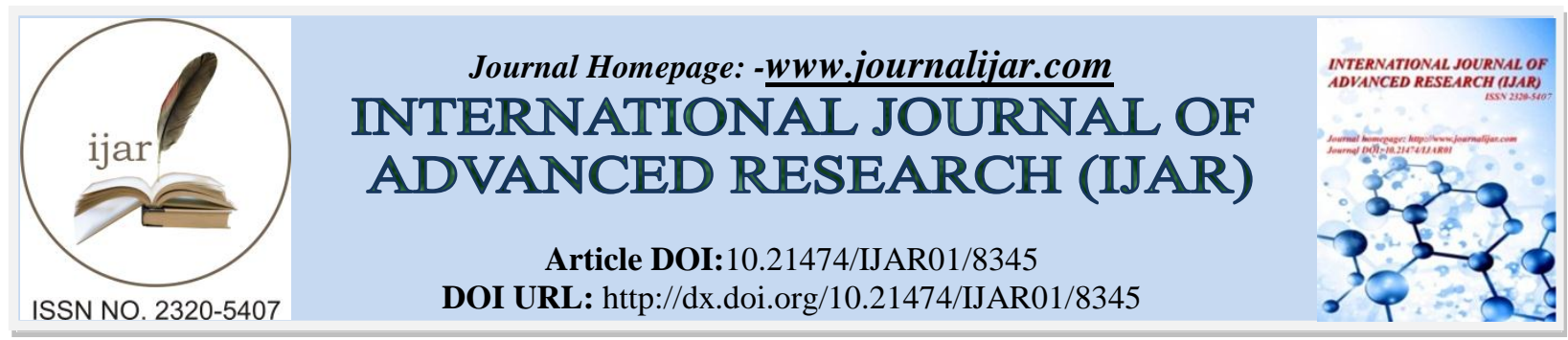

RESEARCH ARTICLE

\title{
LE SYNDROME DE PRADER WILLI A L'AGE DE L'ADOLESCENCE : DIFFICULTES DE PRISE EN CHARGE : A PROPOS DE DEUX CAS.
}

Nassiba Elouarradi , Ghizlane Elmghari and Nawal Elansari.

Service D'endocrinologie, Diabetologie Et Maladies Metaboliques, Chu Mohammed Vi, Hopital Errazi, Marrakech.

\section{Manuscript Info}

Manuscript History

Received: 08 November 2018

Final Accepted: 10 December 2018

Published: January 2019

Key words:-

Prader Willi, adolescence, genetics.

\begin{abstract}
Prader-Willi syndrome (PWS) is a rare disease first described in 1956. It is an important part of mental retardation related to a genetic cause and is one of the leading causes of syndromic obesity in children. It is responsible for physical, psychological and social disabilities of a particular gravity. The diagnosis is evoked on clinical elements and confirmed only by genetic analysis. The management of this disease is multidisciplinary and can be extremely difficult in the teenage age due to behavioral disorders and the total lack of autonomy of patients.

We report the case of two patients followed in our training for the treatment of Prader Willi syndrome in adolescence with severe behavioral disorders
\end{abstract}

Copy Right, IJAR, 2017,. All rights reserved.

\section{Introduction:-}

Le syndrome de Prader-Willi (SPW) est une maladie génétique complexe due à un défaut d'expression des gènes de la région du chromosome 15q11-q13 d'origine paternelle. Il associe : une hypotonie néonatale sévère, l'apparition précoce d'une obésité morbide associée à une hyperphagie, une petite taille, un hypogonadisme, des problèmes de comportement et des troubles psychiatriques et d'apprentissage [1]. Il existe différentes formes génétiques de la maladie dont les principales sont la délétion et la disomie uniparentale maternelle. Vue la diversité des manifestations de cette maladie, la nécessité d'une prise en charge précoce, globale s'impose. Ces différentes compétences sont à associer en fonction des enfants, de leur âge et du contexte clinique .Cela a pour conséquence non seulement d'améliorer la qualité de vie des patients mais aussi de diminuer les difficultés rencontrées par les familles et les soignants en mettant en place un accompagnement de l'entourage[2].

\section{Observations:-}

Il s'agit d'un patient âgé de 18 ans , issu d'un mariage consanguin premier degré, ayant comme antécédents une souffrance néonatale avec antécédent d'hospitalisation à $\mathrm{J} 40$ de vie pour refus de tétée, hypotrophie et hypotonie avec notion de retard des acquisitions psychomotrices : Position assise à 1 an, langage et propreté acquisesà 3 ans et jamais scolarisé. Il est également connu diabétique depuis 8 ans sous insuline. La mère apporte la notion de troubles de conduites alimentaires type polyphagie depuis l'enfance avec notion de troubles de comportements type agitation et agressivité à l'âge de 16 ans nécessitant une prise en charge par un psychologue et mise sous traitement antipsychotique. L'examen clinique objective un patient présentant des yeux en amande avec une malformation du pied gauche en adductus, un poids à $115 \mathrm{~kg}(+3 \mathrm{DS})$, une taille à $162 \mathrm{~cm}$ (entre - 2,et $-3 \mathrm{DS})$, un tour de taille à $124 \mathrm{cms}$, un indice de masse corporelle à $43,81 \mathrm{~kg} / \mathrm{m} 2$, stade Tanner : P2, bourses vides et taille de la verge à $4 \mathrm{cms}$

Corresponding Author:-Nassiba Elouarradi.

Address:-Service D’endocrinologie, Diabetologie Et Maladies Metaboliques, Chu Mohammed Vi, Hopital Errazi, Marrakech. 
(inf à -2 DS), Le bilan a objectivé un hypogonadisme hypogonadotrope, un foie stéatosique à l'échographie abdominale et une TDM abdomino-pelvienne n'objectivant pas de gonades.

Le deuxième patient est un adolescent âgé de 16 ans ayant comme antécédents la notion de refus de tétée et d'hypotonie nécessitant une hospitalisation à $\mathrm{J} 7$ de vie avec notion de retard des acquisitions psychomotrices : un retard de la marche jusqu'à l'âge de 3 ans avec retard de languagejusqu' à l'âge de 11 ans . La mère rapporte également des troubles de comportements type agitation et agressivité avec troubles alimentaires : Polyphagie avec vol de la nourriture de ses camarades et ses frères. Patient mis depuis 2 ans sous neuroleptiques et dépakine. L'examen clinique objective une taille à $154 \mathrm{~cm}$ (entre -2 et $-3 \mathrm{DS})$, un poids à $121 \mathrm{~kg}(>+3 \mathrm{DS})$ avec un IMC à $51,02 \mathrm{~kg} / \mathrm{m} \mathrm{kg} / \mathrm{m}^{2}$ et tour de taille à $133 \mathrm{~cm}$. L'examen des organes génitaux externes a objectivé une cryptorchidie bilatérale, avec un micropénis enfouis de $0,5 \mathrm{~cm}$, le bilan hormonal a objectivé un hypogonadisme hypogonadotrope avec un taux de testostérone à $0,12 \mathrm{ug} / 1$, et une hypothyroïdie périphérique :TSHus: 8,1 mUI/1 , T41 : 11,64 pmol/1 (12- $22 \mathrm{pmol} / \mathrm{l})$. Le patient a été mis sous 1-thyroxine.

Le diagnostic a été confirmé par l'étude génétique dans les deux cas .La prise en charge a consisté en une prise en charge diététique et psychologique avec la recherche et traitement des complications de l'obésité.

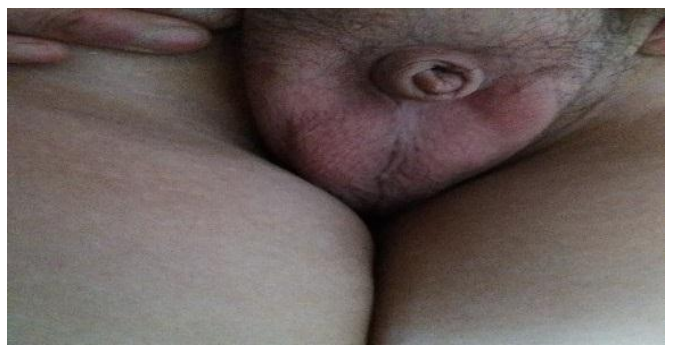

Figure 1:-micropénis avec verge enfouie avec cryptorchidie bilatérale.

\section{Discussion:-}

Le syndrome de Prader-Willi (SPW) est une maladie génétique rare, décrite pour la première fois en 1956 par Prader, Labbhart et Willi [3] qui se caractérise par un dysfonctionnement hypothalamo-hypophysaire associé à une hypotonie majeure pendant la période néonatale et les deux premières années de vie ; puis de l'enfance à l'âge adulte, les problèmes principaux sont l'apparition d'une hyperphagie avec le risque d'obésité morbide, des difficultés d'apprentissage et des troubles du comportement, voire des troubles psychiatriques majeurs. Le dénominateur commun des différentes anomalies génétiques du SPW est l'absence de contribution des gènes de la région 15q11q13 d'origine paternelle au patrimoine héréditaire de l'enfant atteint. L'absence de l'expression de cette partie du chromosome 15 d'origine paternelle est responsable du SPW[4,5].

Beaucoup des troubles rencontrés dans le SPW semblent être en relation avec un dérèglement de la fonction hypothalamique : trouble de la satiété, retard de croissance, hypogonadisme central, troubles du comportement, trouble de la régulation thermique. Cependant, les méthodes d'imagerie cérébrale n'ont pas mis en évidence d'anomalies majeures en dehors d'une diminution de la taille de l'antéhypophyse parfois retrouvée à l'IRM[6].L'hypogonadisme, comme chez nos deux patients, entraîne chez le garçon une cryptorchidie et beaucoup plus rarement un micropénis $(6 \%)[7,8,9]$.

Le déficit intellectuel est rarement majeur et est extrêmement variable d'un enfant à l'autre (QI moyen de 60) et il est souvent associé à des difficultés d'apprentissage $[10,11]$.Les tableaux psychiatriques à type de névroses ou psychoses sont rares chez l'enfant. En revanche, il existe des troubles du comportement qui sont variés et non spécifiques à type de : crises brutales de colère incontrôlable survenant souvent à l'occasion de frustrations mineures pouvant aboutir à des violences physiques contre l'entourage ou à la destruction d'objets environnants, comportements obsessionnels avec répétitions verbales, et tendance dépressive. Comme pour toutes les autres atteintes, il existe une extrême variabilité du tableau clinique psychiatrique [12,13]. Récemment, des lésions cérébrales de la substance blanche ont été mises en évidence dans une étude portant sur 17 sujets présentant un SPW, âgés de 0,9 à 39 ans : seuls les sujets âgés de plus de 18 ans présentent ces anomalies [14] 
La prise en charge diététique et hormonale doit être couplée à une prise en charge multidisciplinaire globale associant : la psychothérapie, la kinésithérapie ,l'orthophonie et la psychomotricité.

Cette prise en charge nécessite une implication familiale importante surtout à l'âge de l'adolescence car ce sont le plus souvent les troubles du comportement qui empêchent la scolarisation normale et l'insertion sociale. Les troubles psychiatriques doivent être pris en charge sur le plan médicamenteux en asseyant d'utiliser au maximum de faibles doses.

\section{Sur le plan diététique:-}

Il est conseillé d'étendre le mode d'alimentation à l'ensemble de la famille afin d'éviter les frustrations, limiter les sources de troubles du comportement et d'accès de colère. La personne atteinte d'un SPW ne peut gérer seul son problème alimentaire car la faim reste incontrôlable. Il devient parfois nécessaire de rendre inaccessible tout accès à la nourriture.

\section{Sur le plan endocrinien:-}

Des études ont permis l'obtention en 2000, dans le cadre de la procédure spéciale pour les médicaments orphelins, de l'autorisation de mise sur le marché (AMM) de l'hormone de croissance comme traitement du SPW de l'enfant dans le but d'améliorer la composition corporelle et/ou la croissance. Ce traitement a considérablement modifié l'évolution staturopondérale de ces enfants [15]. À la fin de la croissance il est conseillé de réaliser une évaluation comme chez les enfants traités pour un déficit en GH et une transition avec les services d'adulte doit être mise en place afin d'éviter l'interruption du suivi. À l'heure actuelle il n'y a pas d'indication pour le traitement par GH à l'âge adulte [16]. Chez le garçon le traitement par gonadotrophines chorioniques ne permet pas un développement pubertaire satisfaisant [11]. Le traitement par testostérone est plus simple et s'administre par voie intramusculaire toutes les trois semaines comme dans les autres hypogonadismes (doses progressives sous contrôle de la testostéronémie). Certains cas d'agressivité accrue sous traitement ont été rapportés mais cela reste à confirmer.

\section{Conclusion:-}

Il est nécessaire de mettre en place une prise en charge globale et multidisciplinaire pour les patients atteints du SPW comme ce que nous avons rapporté pour nos deux patients. Le diagnostic et la prise en charge précoces ainsi que l'utilisation d'hormone de croissance ont transformé leurs qualité de vie cependant il n'y a pas encore de recul sur l'effet du traitement à l'âge adulte, en particulier sur les troubles du comportement et l'autonomie. Chez l'adulte, les complications liées à la maladie et essentiellement à l'obésité ainsi que les difficultés d'autonomie, restent encore très importantes.

\section{References:-}

1. Maïthé Tauber, Gwenaëlle Diènea, Mélanie Glattard, EricBieth (2006) : Le syndrome de Prader Willi : mt pédiatrie, vol. $9, \mathrm{n}^{\circ} 4$, juillet-août .

2. Bachère N, Diene G, Moulin P, Tauber M (2004): Evolution of young children with Prader Willi syndrome diagnosed during the first twomonths of life and managed by a multidisciplinary team. 43th annualmeeting for the European Society for Pediatric Endocrinology. Basel.

3. Prader A, Labhart A, Willi H (1956) Ein Syndrom von Adipositas, Kleinwuchs, Kryptoorchismus und Oligophrenie nach Myatonieartigem Zustand im Neugeborenenalter. Schweiz Med Wschr 86:1260-1261

4. Nicholls RD, Knoll JH, Butler MG, Karam S, Lalande M (2004). Genetic imprinting suggested by maternal heterodisomy in nondeletion Prader-Willi syndrome. Nature 1989;342:281-285.

5. Goldstone AP (2004) . Prader-Willi syndrome: advances in genetics, pathophysiology and treatment. Trends Endocrinol Metab;15:12-20.

6. G. Diene , A. Postel-Vinay, G. Pinto , M. Polak, M. Tauber (2007). Le syndrome de Prader Willi. Annales d'EndocrinologieVol 68, N²-3 - pp. 129 137Doi10.1016/j.ando.2007.03.002

7. Crino A, Schiaffini R, Ciampalini P, et al (2003). Hypogonadism and pubertal development in Prader-Willi syndrome. Eur J Pediatr;162:327-333.

8. Eiholzer U, l'Allemand D, Rousson V, et al (2006). Hypothalamic and gonadal components of hypogonadism in boys with Prader-Labhart-Willi syndrome. J Clin Endocrinol Metab;91:892-898.

9. Craig ME, Cowell CT, Larsson P, et al (2006). Growth hormone treatment and adverse events in Prader-Willi syndrome: data from KIGS (the Pfizer International Growth Database). Clin Endocrinol (Oxf) ;65:178-185. 
10. Whittington J, Holland A, Webb T, Butler J, Clarke D, Boer H (2004). Cognitive abilities and genotype in a population-based sample people with Prader-Willi syndrome. J Intellect Disabil Res ;48:172-187.

11. Whittington J, Holland A, Webb T, Butler J, Clarke D, Boer H (2004). Academic underachievement by people with Prader-Willi syndrome. J Intellect Disabil Res ;48:188-200.

12. Whittington J, Holland T (2004). Prader-Willi syndrome: development and manifestations. Cambridge: University Press.

13. Boer H, Holland A, Whittington J, Butler J, Webb T, Clarke D (2002). Psychotic illness in people with Prader Willi syndrome due to chromosome 15 maternal uniparental disomy. Lancet ;359:135-136.

14. Miller J, Kranzler J, Liu Y, et al (2006). Neurocognitive findings in Prader-Willi syndrome and early-onset morbid obesity. J Pediatr ;149:192-198.

15. Tauber M, Barbeau C, Jouret B, et al (2000). Auxological and endocrine evolution of 28 children with PraderWilli syndrome: effect of GH therapy in 14 children. Horm Res ;53:279-287.

16. Hoybye C, Hilding A, Jacobson H, Thoren M (2002). Metabolic profile and body composition in adults with Prader-Willi syndrome and severe obesity. J Clin Endocrinol Metab ;87:3590-3597.

17. Eiholzer U, l'Allemand D, Rousson V, et al (2006). Hypothalamic and gonadal components of hypogonadism in boys with Prader-Labhart-Willi syndrome. J Clin Endocrinol Metab;91:892-898. 